\title{
Performance Comparison of Wavefront-Sensorless Adaptive Optics Systems by Using of the Focal Plane
}

\author{
Huizhen Yang, ${ }^{1}$ Zhen $\mathrm{Zhang}^{2}$ and Jian $\mathrm{Wu}^{2}$ \\ ${ }^{1}$ School of Electronic Engineering, Huaihai Institute of Technology, Lianyungang 222005, China \\ ${ }^{2}$ School of Information and Electrical, China University of Mining and Technology, Xuzhou 221008, China \\ Correspondence should be addressed to Huizhen Yang; yanghz526@126.com
}

Received 6 November 2014; Accepted 19 December 2014

Academic Editor: Nicusor Iftimia

Copyright (c) 2015 Huizhen Yang et al. This is an open access article distributed under the Creative Commons Attribution License, which permits unrestricted use, distribution, and reproduction in any medium, provided the original work is properly cited.

\begin{abstract}
The correction capability and the convergence speed of the wavefront-sensorless adaptive optics (AO) system are compared based on two different system control algorithms, which both use the information of focal plane. The first algorithm is designed through the linear relationship between the second moment of the aberration gradients and the masked far-field intensity distribution and the second is stochastic parallel gradient descent (SPGD), which is the most commonly used algorithm in wavefront-sensorless AO systems. A wavefront-sensorless AO model is established with a 61-element deformable mirror (DM) and a CCD. Performance of the two control algorithms is investigated and compared through correcting different wavefront aberrations. Results show that the correction ability of AO system based on the proposed control algorithm is obviously better than that of AO system based on SPGD algorithm when the wavefront aberrations increase. The time needed by the proposed control algorithm is much less than that of SPGD when the AO system achieves similar correction results. Additionally, the convergence speed of the proposed control algorithm is independent of the turbulence strength while the number of intensity measurements needed by SPGD increases as the turbulence strength magnifies.
\end{abstract}

\section{Introduction}

Compared with the conventional adaptive optics $(\mathrm{AO})$ system [1], the complexity of wavefront-sensorless AO system [2] is reduced greatly because of no wavefront sensor in system structure. The most significant potential advantage of this kind of $\mathrm{AO}$ system is related to the fact that the farfield intensity distribution can be used as the feedback signal. The wavefront-sensorless AO system can be divided into two categories according to control algorithms, one is model-free $\mathrm{AO}$ systems and the other is model-based $\mathrm{AO}$ systems. The former directly uses various blind optimization algorithms as the control algorithm of the system, such as the stochastic parallel gradient descent algorithm (SPGD-stochastic parallel gradient descent) [3, 4], Alopex algorithm [5], simulated annealing [6], and genetic algorithm [7]. Model-based wavefront-sensorless $\mathrm{AO}$ technology often makes use of some kind of model to design the control algorithm for system, such as modal method $[8,9]$, the nonlinear optimization method [10], or geometric optical principles [11].
In this paper, we design a control algorithm for the wavefront-sensorless $\mathrm{AO}$ system through the linear relationship between the second moment of the aberration gradients and the masked far-field intensity distribution. An AO system model is established with a 61-element deformable mirror (DM) and a CCD. The correction capability and the convergence speed of the $\mathrm{AO}$ system are investigated and compared with SPGD control algorithm, which is the most commonly used control algorithm for wavefront-sensorless AO systems and verified to be relatively fast control method [12].

\section{Control Algorithm Based on the Linear Relationship between the Second Moment of the Aberration Gradients and the Masked Far-Field Intensity Distribution}

2.1. Theory Basis of Control Algorithm. The centroid of the far-field intensity distribution in geometric optics is related 
to the aberration of the input wavefront. A linear relationship between the second moment of the aberration gradients and the masked far-field intensity distribution can be achieved through a certain mathematical transformation [11]:

$$
\mathrm{SM} \approx c_{0}(1-\mathrm{MDS})
$$

where $\mathrm{SM}$ is the second moment of the aberration gradients and $c_{0}$ is a constant, the slope of the trend line, which is determined by the detector radius $R$. MDS is defined as follows:

$$
\operatorname{MDS}=\frac{\iint I\left(x^{\prime}, y^{\prime}\right)\left[1-r^{2} / R^{2}\right] d x^{\prime} d y^{\prime}}{\iint I\left(x^{\prime}, y^{\prime}\right) d x^{\prime} d y^{\prime}}
$$

where $I\left(x^{\prime}, y^{\prime}\right)$ is the far-field intensity distribution and 1 $r^{2} / R^{2}\left(r=\sqrt{x^{\prime 2}+y^{\prime 2}}\right)$ is the mask. In practice, the mask could be implemented by the weighting of pixels within the software when the photodetector is replaced by a CCD camera.

In order to find out the control parameters of deformable mirror, $N$ orthogonal modes are taken as predetermined bias functions and are added by the DM sequentially with coefficient $\alpha$ to the wavefront aberration to be corrected. Then the detector measurements are recorded and the $N \operatorname{MDS}_{i}(i=$ $1, \ldots, N)$ are calculated according to (2). Control parameters $V$ can be estimated by

$$
V \approx \frac{S^{-1}\left(c_{0} M-\alpha^{2} S_{m}\right)}{2 \alpha},
$$

where

$$
M=-\left\{\begin{array}{c}
\mathrm{MDS}_{1}-\mathrm{MDS}_{0} \\
\mathrm{MDS}_{2}-\mathrm{MDS}_{0} \\
\vdots \\
\mathrm{MDS}_{N}-\mathrm{MDS}_{0}
\end{array}\right\}
$$

and $\mathrm{MDS}_{0}$ is the corresponding MDS of the wavefront aberration to be corrected. The matrix $S$ is the second moment of the wavefront gradients, which can be calculated by the bias function. The vector $S_{m}$ is the diagonal vector of matrix $S$. $S$ can be calculated according to the following equation, where $F(x, y)$ is the predefined bias function:

$$
\begin{aligned}
S(i, j)=D^{-1} \int_{D} & {\left[\frac{\partial}{\partial x} F_{i}(x, y) \frac{\partial}{\partial x} F_{j}(x, y)\right] } \\
+ & {\left[\frac{\partial}{\partial y} F_{i}(x, y) \frac{\partial}{\partial y} F_{j}(x, y)\right] d x d y . }
\end{aligned}
$$

2.2. Control Algorithm Design. A set of bias functions are defined to represent wavefront aberrations. We use Zernike polynomial as the bias function. The wavefront to be corrected $\phi(x, y)$ can be written as a linear combination of the Zernike polynomial $Z(x, y)$ :

$$
\phi(x, y)=\sum_{i=1}^{N} \beta_{i} Z_{i}(x, y),
$$

where $N$ is the Zernike order number, $\beta_{i}$ is the Zernike coefficient of the $i$ th-order Zernike mode, and $Z_{i}(x, y)$ is the $i$ thorder Zernike mode. The phase compensation $u(x, y)$, introduced by DM, can be decomposed into the superposition of the response functions of actuators linearly:

$$
u(x, y)=\sum_{j=1}^{K} v_{j} V_{j}(x, y)
$$

where $v_{j}$ is the control signal, $V_{j}(x, y)$ is the response function of the $j$ th actuator, and $K$ is the number of DM actuator. Using DM to fit the wavefront aberration described by Zernike polynomials, we can get

$$
\phi(x, y)=u(x, y)+e(x, y)
$$

where $e(x, y)$ is the residual wavefront of the DM fitting. Equation (8) can be changed into the following equation after $\phi(x, y)$ and $u(x, y)$ are replaced by (6) and (7), respectively:

$$
\sum_{i=1}^{N} \beta_{i} Z_{i}(x, y)=\sum_{j=1}^{K} v_{j} V_{j}(x, y)+e(x, y) .
$$

Both sides of (9) are multiplied by one influence function $V_{l}(x, y)$ at the same time and the integral in the aperture $D$ is calculated:

$$
\begin{aligned}
& D^{-1} \int_{D} \sum_{i=1}^{N} \beta_{i} Z_{i}(x, y) V_{l}(x, y) d x d y \\
& \quad=D^{-1} \int_{D}\left[\sum_{j=1}^{K} v_{j} V_{j}(x, y)+e(x, y)\right] V_{l}(x, y) d x d y .
\end{aligned}
$$

Equation (10) can get a matrix equation:

$$
C_{v z} \beta=C_{v} v+\varepsilon
$$

where $C_{v z}$ is the cross-correlation matrix with size $K \times N$ between the Zernike mode $Z(x, y)$ and the influence function $V(x, y), C_{v}$ is the coupling matrix between different influence function $V(x, y)$ with size $K \times K, \beta$ is the Zernike coefficient with size $N$, and $v$ is the control voltage vector with size $K$. $C_{v z}(i, j)$ and $C_{v}(i, j)$ have the following form, respectively:

$$
\begin{gathered}
C_{v z}(i, j)=D^{-1} \iint_{D} V_{i}(x, y) Z_{j}(x, y) d x d y \\
i=1, \ldots, K, \quad j=1, \ldots, N \\
C_{v}(i, j)=D^{-1} \iint_{D} V_{i}(x, y) V_{j}(x, y) d x d y \quad i, j=1, \ldots, K .
\end{gathered}
$$

We can obtain the minimum variance solution of (10):

$$
v^{*}=C_{v}^{-1} C_{z v} \beta .
$$

Equation (13) provides a minimum variance solution to the control voltage vector $v$. 
The control algorithm is divided into two parts: the preprocessing step and the iteration step. The detailed descriptions are as follows.

\section{Preprocessing Step}

(1) Calculate the second moment of the wavefront gradients $S$, the diagonal vector $S_{m}$, and its inverse matrix $S^{-1}$ according to the predefined bias function $Z(x, y)$ by (5).

(2) Measure the influence functions $V(x, y)$ of the DM and compute the cross-correlation matrix $C_{z v}$, the coupling matrix $C_{v}$, and its inverse $C_{v}^{-1}$ according to (12).

Notably, the vector $S_{m}$ and the matrices $S, S^{-1}, C_{v}, C_{v}^{-1}$, and $C_{z v}$ in the preprocessing step can be obtained in advance and these matrixes have nothing to do with the wavefront to be corrected. The above data can be regarded as known after $\mathrm{DM}$ and the bias functions are determined and are imported directly during correcting the wavefront aberrations.

Iterative Step

(1) Design the mask through the programming software.

(2) Gather the corresponding far-field intensity of the wavefront to be corrected $\phi(r)$ from CCD, calculate MDS according to (2), and call it $\mathrm{MDS}_{0}$.

(3) Calculate the corresponding voltage vector of each Zernike mode according to (5) by substituting $a=$ $\left\{0, \ldots, a_{i}, 0, \ldots\right\}$ and apply the voltage vector to the actuators of DM. The wavefront shape introduced by the deformable mirror is superimposed to the wavefront to be corrected $\phi(r)$.

(4) Get the corresponding far-field intensity of the wavefront aberration superimposed from CCD and calculate MDS according to (2). Repeating (3) and (4), we can get the obtained $\mathrm{MDS}_{1}, \mathrm{MDS}_{2}, \ldots, \mathrm{MDS}_{N}$ for $N$ Zernike mode, respectively. The total Zernike coefficient is $a=\left\{\ldots, a_{i}, \ldots, a_{j}, \ldots\right\}$.

(5) Compute $M$ according to (4).

(6) Obtain the corresponding voltage vector $V$ of the wavefront to be corrected $\phi(r)$ according to (3). The voltage vector $V$ is applied to the actuators of $\mathrm{DM}$ through the high voltage amplifier.

(7) Regard the residual wavefront as the wavefront to be corrected $\phi(r)$; repeat (2)-(7) in the iterative step until the algorithm satisfies the termination conditions in advance, such as a certain number of iterations.

The flow chart of the control algorithm is shown in Figure 1, where an inner cycle of control algorithm is considered as one algorithm iteration and we make use of the number of iterations as the end condition of algorithm.

\section{Simulation Platform of Wavefront- Sensorless AO System}

As shown in Figure 2, the wavefront-sensorless AO system consists of a 61-element DM, an imaging lens, a CCD, and

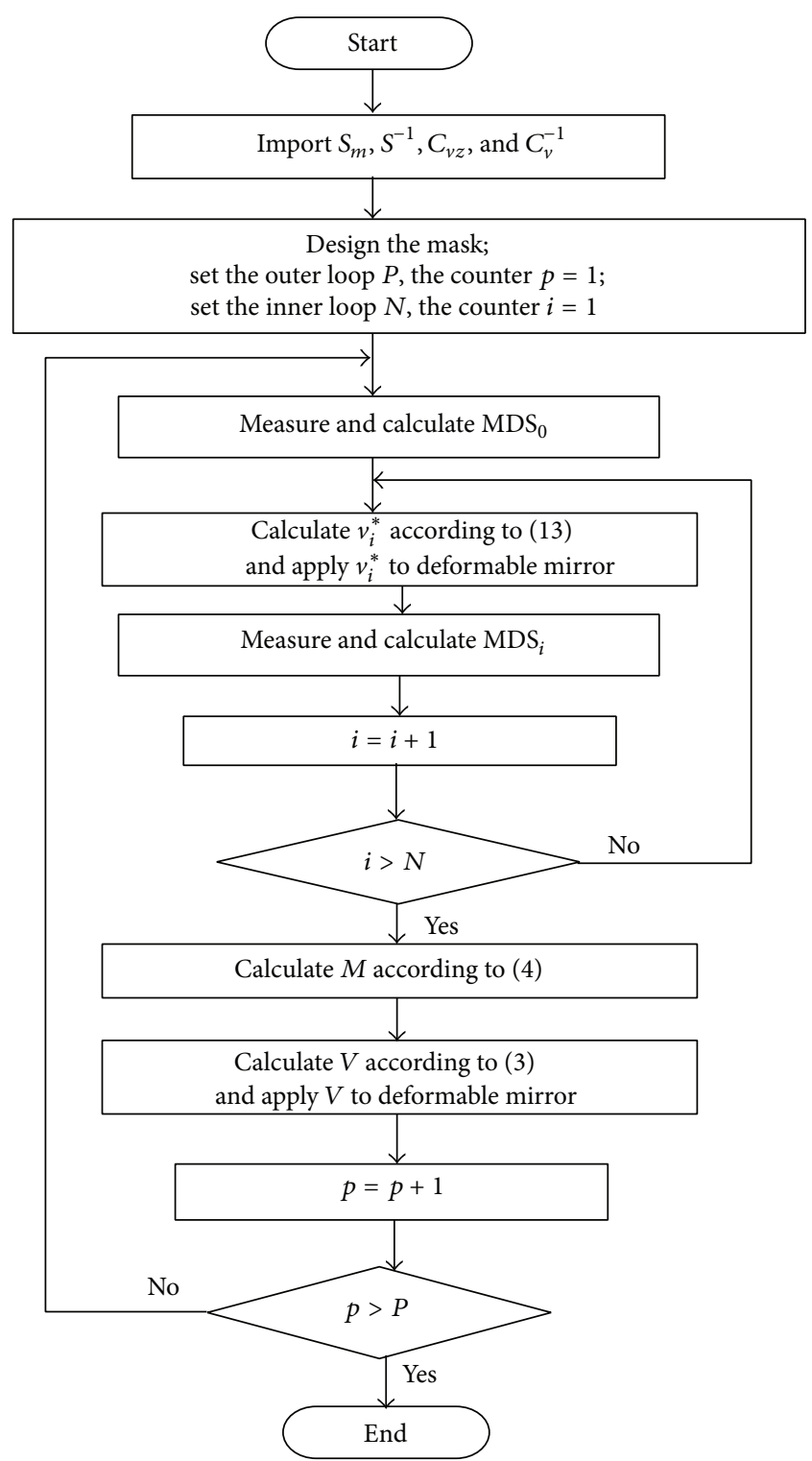

FIGURE 1: The flow chart of control algorithm for wavefront-sensorless adaptive optics system.

the control algorithm. The wavefront to be corrected is reflected to the imaging lens and the imaging lens focuses it into the CCD. The intensity is detected by CCD and provided to the control algorithm. The control algorithm generates a correction voltage vector. The voltage vector is applied to the actuators of 61-element deformable mirror.

On the basis of real measurements, we know that the response function of 61-element deformable mirror actuators is Gaussian distribution approximately [13]. Figure 3 gives the location distribution of 61-element DM actuators. The layout of all actuators is triangular.

The method proposed by Roddier, which makes use of a Zernike expansion of randomly weighted Karhunen-Loeve functions, is used to simulate atmospherically distorted wavefronts [14]. Considering that the low-order aberrations (tilts, defocus, astigmatism, etc.) have the most significant impact 


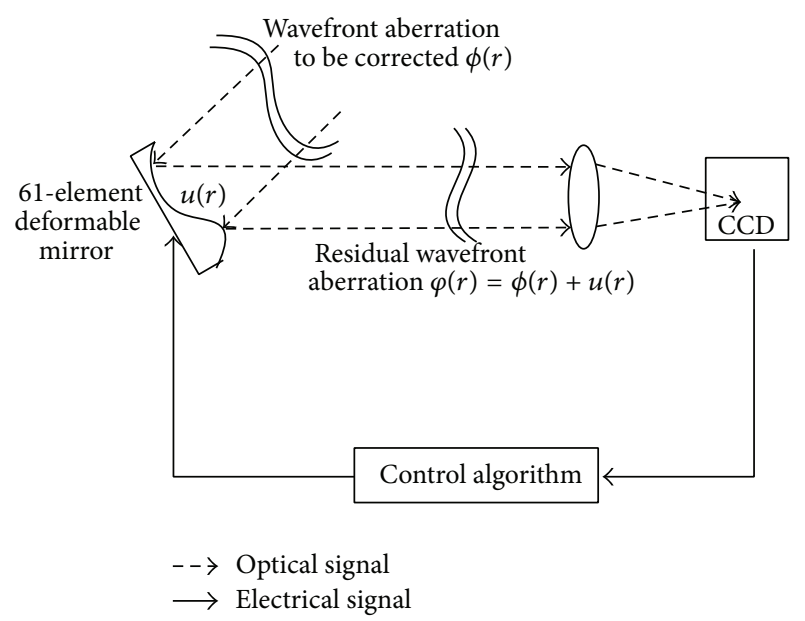

FIGURE 2: Block diagram of wavefront-sensorless adaptive optics system.

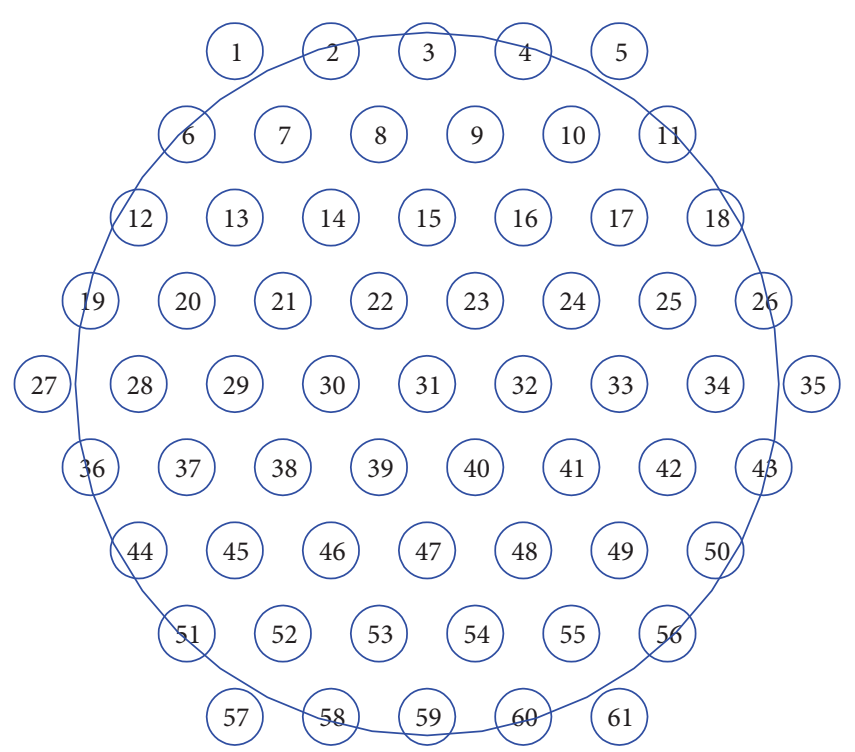

FIgURE 3: Actuators location of 61-element deformable mirror.

on image quality, we use the first 104 Zernike polynomials. The phase screens do not include the tip/tilt aberrations because the tip/tilt aberrations are usually controlled by another control loop and are considered as being removed completely in our simulation. Atmospheric turbulence strength for a receiver system with aperture size $D$ can be characterized by the ratio $D / r_{0}$, where $r_{0}$ is the turbulence coherence length. Phase screens of different atmospheric turbulence strength can be obtained through changing $r_{0}$ in the simulation program.

\section{Results and Analysis}

To investigate the convergence speed and the correction capability of the wavefront-sensorless AO system with a 61element deformable mirror based on the proposed control algorithm, we perform the adaptation process over random phase screens under five different turbulence strengths. The five different turbulence strengths are corresponding to $D / r_{0}=5,10,15,20$, and 25 , respectively. The averaged evolution curves over 500 frames per turbulence strength and the standard deviation evolution curves of the metric are the recorded simulation results. MR (mean radius) of the far-field intensity distribution and SR (Strehl ratio) are used to measure the correction capability of the AO system, respectively. The calculation formula of MR is as follows:

$$
\mathrm{MR}=\frac{\iint_{D} \sqrt{\left(x-x_{0}\right)^{2}+\left(y-y_{0}\right)^{2}} I(x, y) d x d y}{\int I(x, y) d x d y},
$$

where $\left(x_{0}, y_{0}\right)=\int(x, y) I(r) d x d y / \int I(x, y) d x d y$ is the centroid of far-field intensity distribution. The smaller the MR value, the more focused the far-field energy and the smaller wavefront aberration. MR can be easily measured either by a single photodetector with a special mask attached to it or by postprocessing a matrix detector output in the actual system. This measure appears to be the most attractive one as it gives straightforward mathematical meaning to the idea of energy spread. The Strehl ratio (SR) is defined as

$$
\mathrm{SR}=\frac{F[I(x, y)]}{F\left[I_{0}(x, y)\right]}
$$

where $F[\cdot]$ is an operation, which calculates the peak intensity. $I_{0}(x, y)$ is the intensity distribution of ideal plane wavefront.

4.1. Adaptive Process of the Wavefront-Sensorless AO System. The number of inner cycles (iterative steps (3) and (4)) of control algorithm is 102 times because the wavefront aberration is composed of the first 104 Zernike polynomials (excluding tip and tilt) in this paper and an inner cycle of control algorithm is considered as one algorithm iteration. Figures 4(a) and 4(c) show the adaptive process of MR and SR by the control algorithm. Standard deviation (SD) curves of MR over 500 different phase realizations are presented in Figure 4(b). The SD is calculated as follows:

$$
\mathrm{SD}=\frac{\left\langle(\mathrm{MR}-\langle\mathrm{MR}\rangle)^{2}\right\rangle^{1 / 2}}{\langle\mathrm{MR}\rangle} .
$$

From Figures 4(a) and 4(c), we can conclude that averaged curves of different metrics have converged after completing iterations and one iteration can make the $\mathrm{AO}$ system converge approximately. The values of SD of MR are very small after the system converges in Figure 4(b). This shows that the discrete level of MR from the averaged value is very tiny and the control algorithm has strong adaptability to 500 different phase screens.

To compare the correction capability and the convergence speed of AO system, the adaptive process of MR and SR by SPGD algorithm is given in Figure 5. Figure 5 shows that the AO system converges completely after about 1000 iterations under different turbulence strength. 


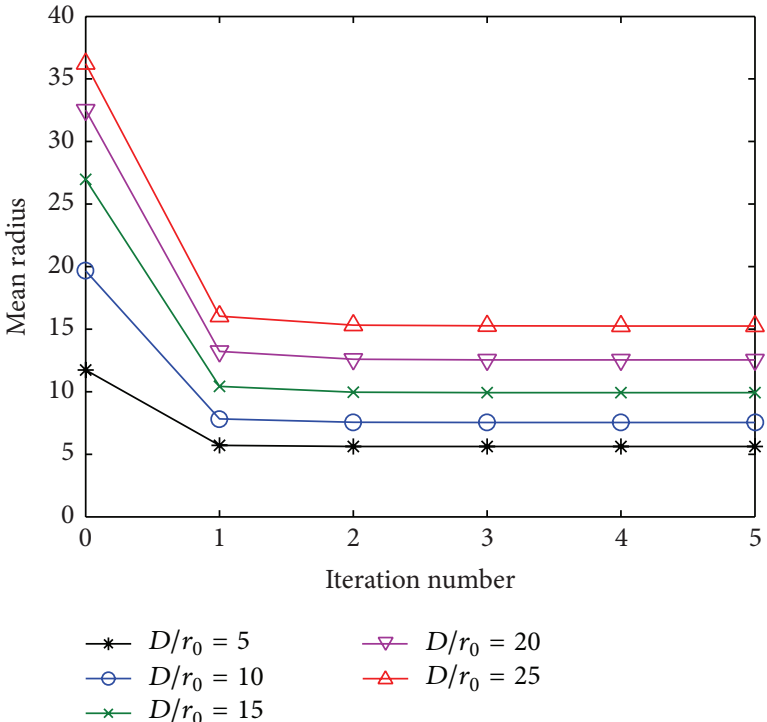

(a)

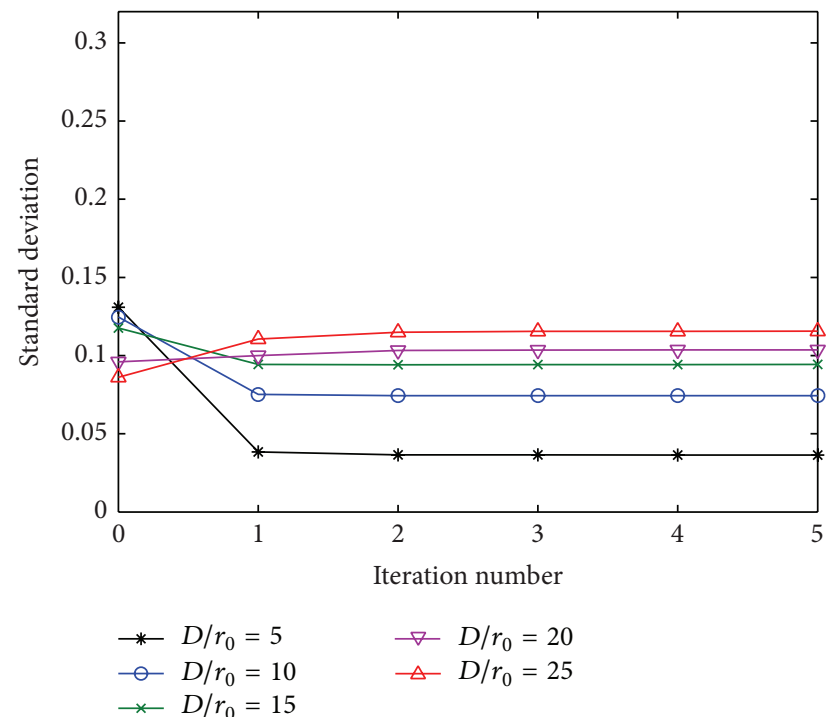

(b)

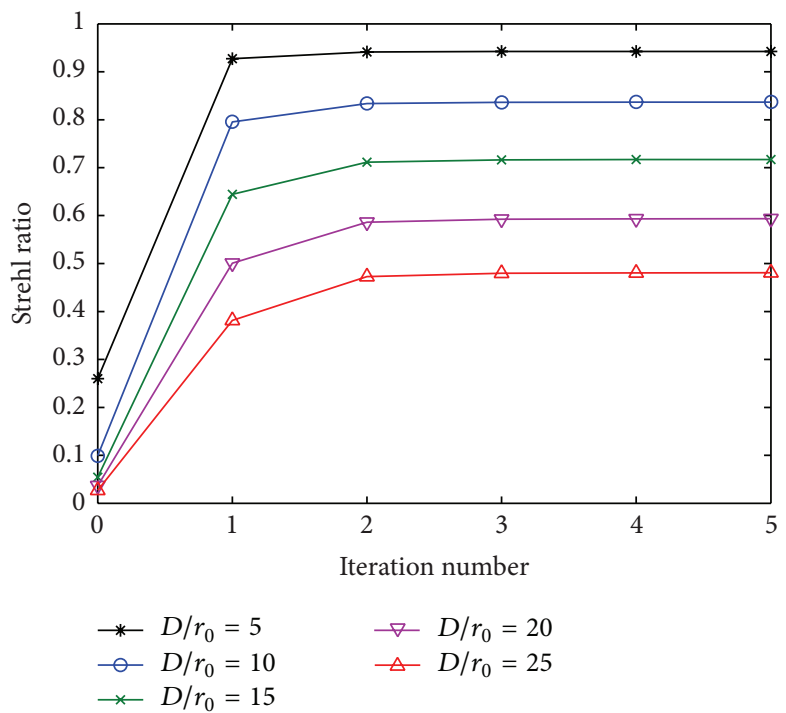

(c)

FIGURE 4: Averaged adaptation process over 500 different phase screens under different turbulence strengths. (a) MR curves, (b) standard deviation curves of MR, and (c) SR curves.

\subsection{Correction Capability Analysis of the Wavefront-Sensorless} AO System. For investigating the correction capability of the wavefront-sensorless AO system based on the proposed control algorithm, we also calculate the averaged MR of the initial far-field intensity distribution and the averaged ideal correction ability of the 61-element deformable mirror over 500 different phase screens. The ideal correction ability can be obtained by the minimum variance solution (4). The corresponding data are given in Figure 6.

The AO system based on two kinds of algorithms has strong correction ability on wavefront aberrations under different turbulence from Figure 6(a), in which MR values are very close to ideal correction of deformable mirror offered by the minimum variance solution. But it can be seen clearly from Figure 6(b) that the correction ability AO system based on SPGD algorithm is worse than that of AO system based on the proposed control algorithm when $D / r_{0}$ is greater than 15 .

\subsection{Convergence Speed Analysis of the Wavefront-Sensorless} AO System. The convergence speed is an important criterion on which the control algorithm can be applied to realtime adaptive optics system. We use the number of intensity measurements to estimate the averaged convergence speed of wavefront-sensorless AO system based on two kinds of control algorithms. The number of intensity measurements can be analyzed from the control algorithm process. The total number of intensity measurements is computed through the times of inner cycle multiplied by that of the outer cycle for 


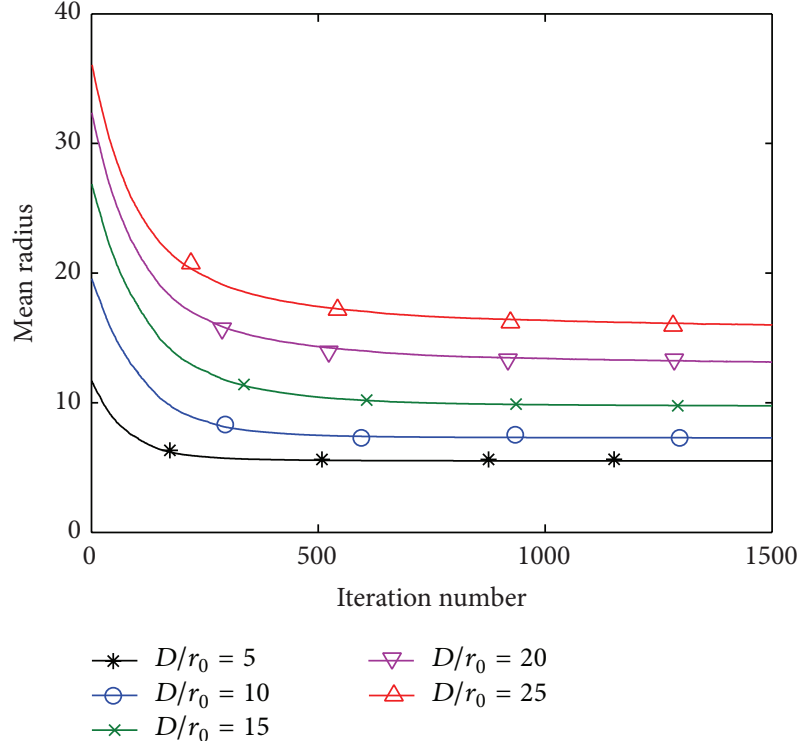

(a)

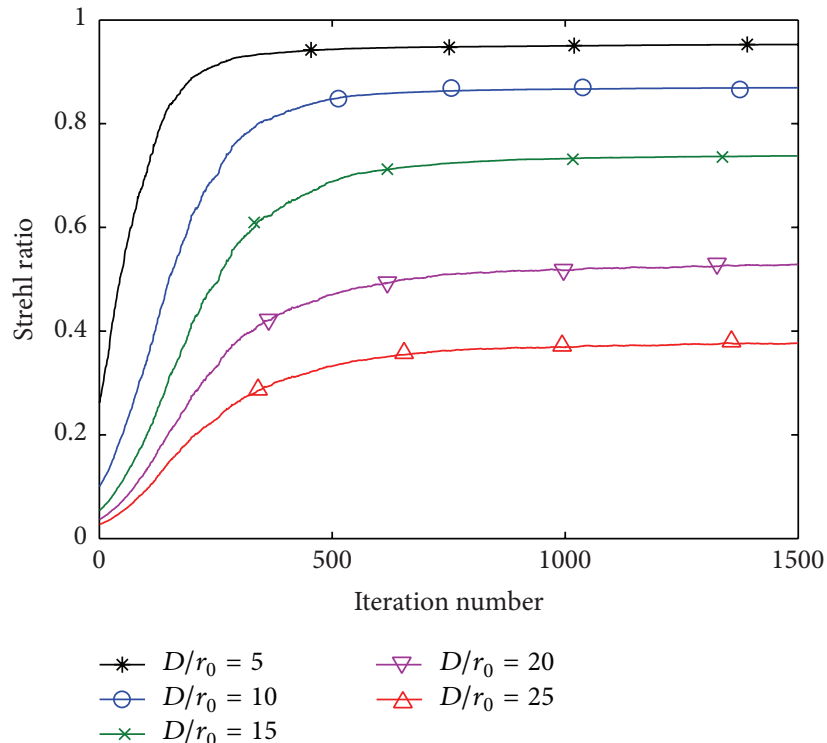

(b)

FIGURE 5: Averaged adaptation process over 500 different phase screens under different turbulence strengths by SPGD algorithm: (a) MR and (b) SR.

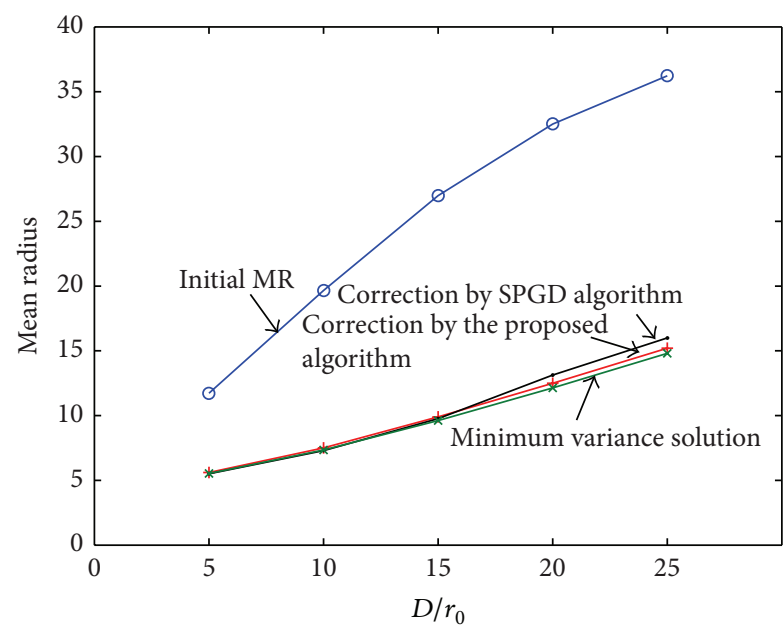

(a)

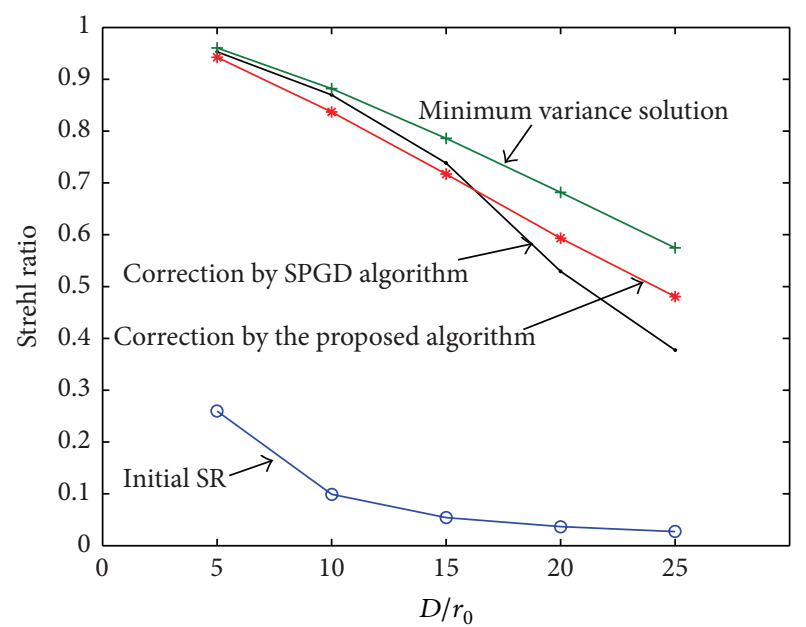

(b)

FIGURE 6: Comparisons of MR of initial, correction by SPGD algorithm, correction by proposed algorithm, and the minimum variance solution (a) and comparisons of SR (b) under different turbulences.

the wavefront-sensorless $\mathrm{AO}$ system based on the proposed algorithm. The number of measurements of $\mathrm{AO}$ system based on SPGD algorithm can be obtained through the times of algorithm iteration multiplied by two because two intensity measurements are needed by one algorithm iteration at least.

From Figure 4(a), we can see that the wavefront-sensorless $\mathrm{AO}$ system based on the proposed algorithm only needs one iteration for algorithm converging, which means the system needs $N+1$ photodetector measurements when $N$-order aberrations are corrected. The system needs 103 intensity measurements in this paper because the wavefront aberration is composed of the first 104 Zernike polynomials (excluding tip and tilt). Figure 4(a) also shows that the number of intensity measurements does not increase as the turbulence strength magnifies.

We can get the times of iteration needed by the wavefrontsensorless AO system based on SPGD from Figure 5 when MR decreases to the value where the proposed method arrives after one iteration and further achieve the number of intensity measurements through multiplying the times of iteration by two. Those data are given in Table 1, where the sign ">" indicates that the value of MR has not been reached at 12.5 and 
TABLE 1: Comparison of the number of intensity measurements of SPGD with that of the proposed method.

\begin{tabular}{lccccc}
\hline$D / r_{0}$ & 5 & 10 & 15 & 20 & 25 \\
\hline SPGD & 826 & 946 & 1458 & $>3000$ & $>3000$ \\
Proposed method & 103 & 103 & 103 & 103 & 103 \\
\hline
\end{tabular}

15.2 on $D / r_{0}=20$, and 25 , respectively, after the $\mathrm{AO}$ system completes 1500 iterations.

From data in Table 1, we can see that the number of intensity measurements needed by $\mathrm{AO}$ system based on SPGD is about 8 times, 9 times, and 14 times that of the wavefront-sensorless $\mathrm{AO}$ system based on the proposed algorithm when $D / r_{0}$ is 5,10 , and 15 , respectively. More intensity measurements are needed by SPGD when $D / r_{0}$ is 20 and 25 . In other words, the time needed by the wavefrontsensorless AO system based on SPGD is far more than that of the wavefront-sensorless AO system based on the proposed algorithm when the $\mathrm{AO}$ system achieves similar correction results. Additionally, the number of intensity measurements needed by SPGD-based AO system increases as the turbulence strength magnifies from the data in Table 1.

\section{Conclusions}

The centroid of the far-field intensity distribution in geometric optics is related to the aberration of the input wavefront. A linear relationship between the second moment of the aberration gradients and the masked far-field intensity distribution can be achieved through certain mathematical transformations. A closed-loop control algorithm is designed by using the linear relationship. An adaptive optics (AO) system simulation platform is established with a 61-element deformable mirror and a CCD. The convergence speed and the correction capability are investigated and compared with stochastic parallel gradient descent (SPGD) control algorithm, the most commonly used control algorithm for wavefront-sensorless adaptive optics systems through correcting wavefront aberrations under different turbulence strength.

Research results show that the control algorithm proposed has strong adaptability to different phase screens. The AO system based on two kinds of algorithms has strong correction ability on wavefront aberrations under different turbulence when the turbulence strength is relatively small. However, the correction ability of AO system based on SPGD algorithm is obviously worse than that of AO system based on the proposed control algorithm when the wavefront aberrations increase. The AO system based on the proposed control algorithm only requires $N+1$ measurements of the far-field spot when $N$ orders aberrations are corrected. The time needed by SPGD-based AO system is far more than that of the wavefront-sensorless AO system based on the proposed control algorithm when the $\mathrm{AO}$ system achieves similar correction results. Additionally, the convergence speed of the wavefront-sensorless $\mathrm{AO}$ system based on the proposed control algorithm is independent of the turbulence strength while the number of intensity measurements needed by SPGD-based AO system increases as the turbulence strength magnifies. Therefore, the control algorithm provided in the paper can greatly improve the convergence speed of the wavefront-sensorless AO systems, which provides the theoretical basis for applications of adaptive optics technology in real-time wavefront correction system.

\section{Conflict of Interests}

The authors declare that there is no conflict of interests regarding the publication of this paper.

\section{Acknowledgments}

This work is supported by Natural Science Foundation of Jiangsu Province of China (Grant no. BK2012653) through Huaihai Institute of Technology and Key Lab Foundation on Adaptive Optics of Chinese Academy of Sciences (Grant no. LAOF 201302).

\section{References}

[1] R. K. Tyson, Principle of Adaptive Optics, CRC Press, New York, NY, USA, 3rd edition, 2010.

[2] R. A. Muller and A. Buffington, "Real-time correction of atmospherically degraded telescope images through image sharpening," Journal of the Optical Society of America A, vol. 64, no. 9, pp. 1200-1210, 1974.

[3] M. A. Vorontsov, G. W. Carhart, M. Cohen, and G. Cauwenberghs, "Adaptive optics based on analog parallel stochastic optimization: Analysis and experimental demonstration," Journal of the Optical Society of America A: Optics and Image Science, and Vision, vol. 17, no. 8, pp. 1440-1453, 2000.

[4] C. Geng, W. Luo, Y. Tan, H. Liu, J. Mu, and X. Li, "Experimental demonstration of using divergence cost-function in SPGD algorithm for coherent beam combining with tip/tilt control," Optics Express, vol. 21, no. 21, pp. 25045-25055, 2013.

[5] M. S. Zakynthinaki and Y. G. Saridakis, "Stochastic optimization for a tip-tilt adaptive correcting system," Computer Physics Communications, vol. 150, no. 3, pp. 274-292, 2003.

[6] S. Zommer, E. N. Ribak, S. G. Lipson, and J. Adler, "Simulated annealing in ocular adaptive optics," Optics Letters, vol. 31, no. 7, pp. 939-941, 2006.

[7] P. Yang, M. Ao, Y. Liu, B. Xu, and W. Jiang, "Intracavity transverse modes controlled by a genetic algorithm based on Zernike mode coefficients," Optics Express, vol. 15, no. 25, pp. 1705117062, 2007.

[8] A. Facomprez, E. Beaurepaire, and D. Débarre, "Accuracy of correction in modal sensor-less adaptive optics," Optics Express, vol. 20, no. 3, pp. 2598-2612, 2012.

[9] M. J. Booth, "Wavefront sensorless adaptive optics for large aberrations," Optics Letters, vol. 32, no. 1, pp. 5-7, 2007.

[10] H. Song, R. Fraanje, G. Schitter, H. Kroese, G. Vdovin, and M. Verhaegen, "Model-based aberration correction in a closedloop wavefront-sensor-less adaptive optics system," Optics Express, vol. 18, no. 23, pp. 24070-24084, 2010.

[11] H. Linhai and C. Rao, "Wavefront sensorless adaptive optics: a general model-based approach," Optics Express, vol. 19, no. 1, pp. 371-379, 2011.

[12] H. Yang and X. Li, "Comparison of several stochastic parallel optimization algorithms for adaptive optics system without 
a wavefront sensor," Optics \& Laser Technology, vol. 43, no. 1, pp. 630-635, 2011.

[13] W. H. Jiang, N. Ling, X. J. Rao, and S. Fan, "Fitting capability of deformable mirror," in Active and Adaptive Optical Systems, vol. 1542 of Proceedings of SPIE, pp. 130-137, 1991.

[14] N. Roddier, "Atmospheric wavefront simulation using Zernike polynomials," Optical Engineering, vol. 29, no. 10, pp. 1174-1180, 1990. 

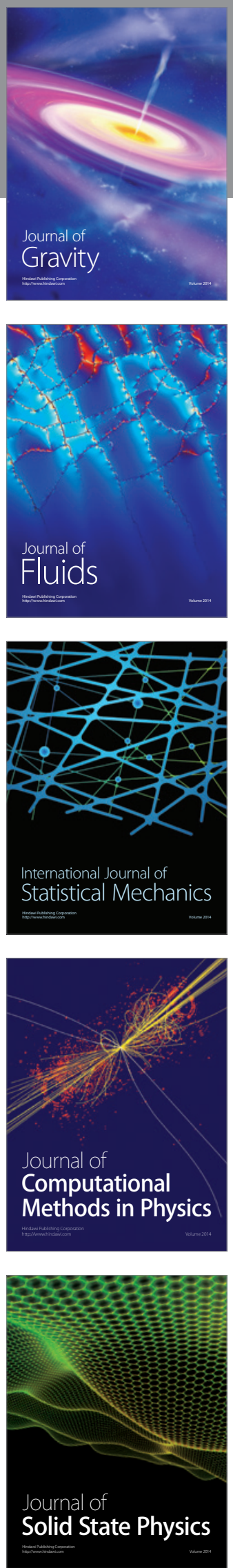

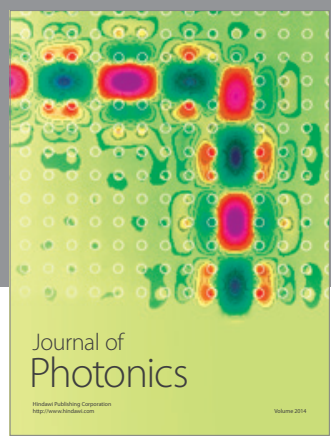

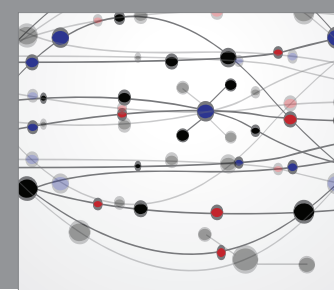

The Scientific World Journal

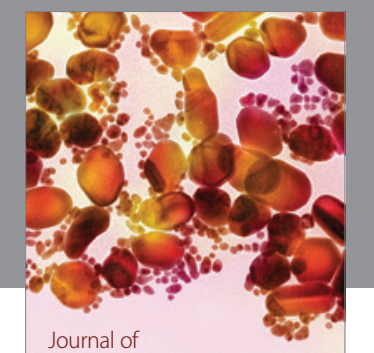

Soft Matter
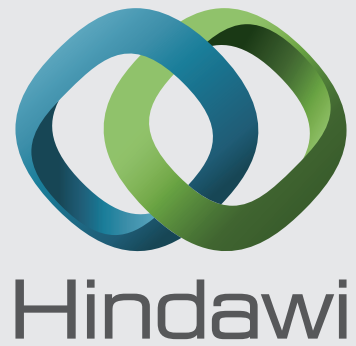

Submit your manuscripts at

http://www.hindawi.com
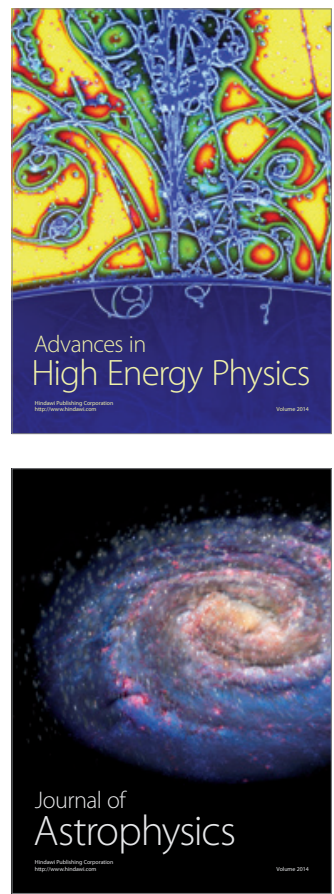
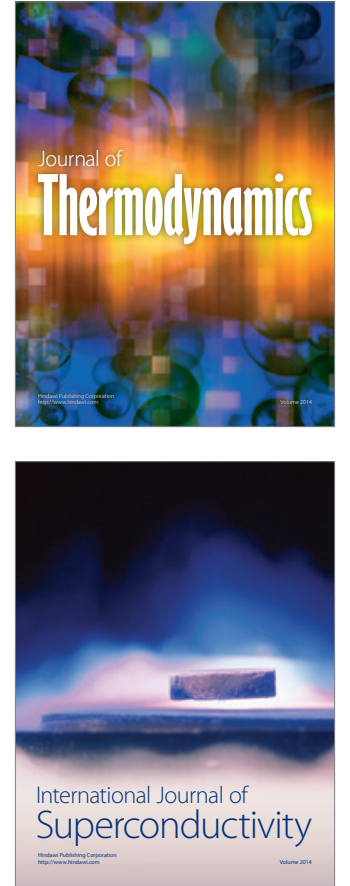
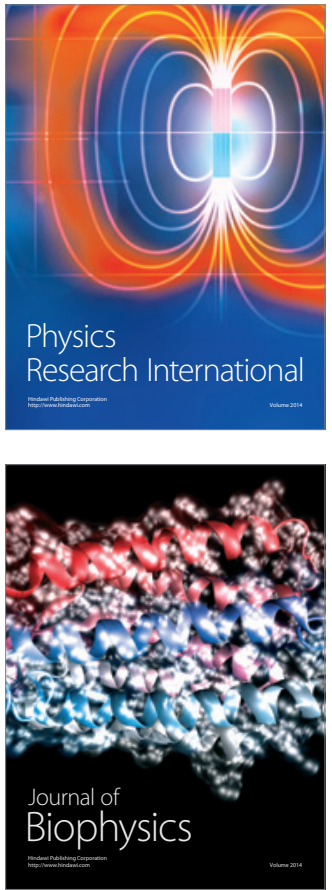
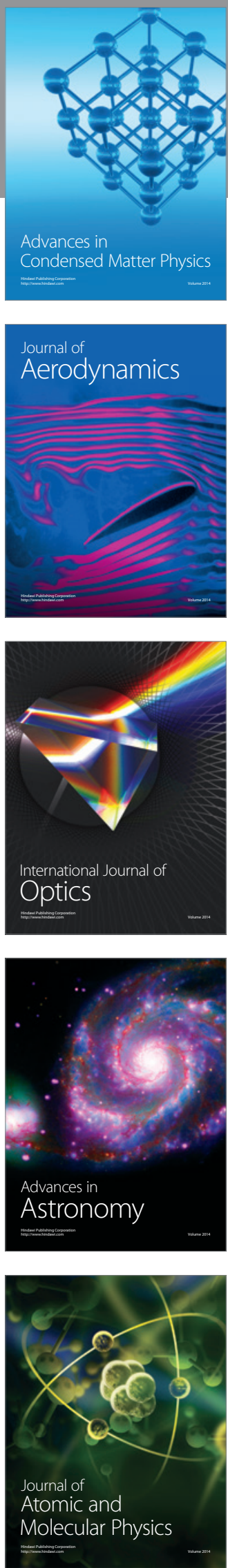